\title{
Hamiltonian cycles in torical lattices
}

\author{
Vladimir K. Leontiev ${ }^{1 \dagger}$
}

${ }^{1}$ Dorodnitsyn Computing Center of RAS, \& Vavilova, 40, Moscow, 117967, Russia

We establish sufficient conditions for a toric lattice $T_{m, n}$ to be Hamiltonian. Also, we give some asymptotics for the number of Hamiltonian cycles in $T_{m, n}$.

Keywords: Hamiltonian cycle, toric lattice, Hardy-Littlewood method.

Let $T_{m, n}=J_{m} \times J_{n}$ be a toric lattice, i.e., the Cartesian product of two directed cycles lengths $m$ and $n$ respectively.

Erdös problem [1]. When $T_{m, n}$ contains Hamiltonian cycles?

The next theorem was proved by A.A.Evdokimov [2].

Theorem $1 T_{m, n}$ is Hamiltonian iff there are solutions of the following Diophantine system

$$
\begin{aligned}
& x+y=\operatorname{gcd}(m, n), \\
& \operatorname{gcd}(x, m)=1, \operatorname{gcd}(y, n)=1
\end{aligned}
$$

(gcd means the greatest common divisor).

Let $J_{m, n}$ be the number of solutions of the system (1). We obtain estimates for $J_{m, n}$ in two special cases. Let

$$
m=\prod_{i=1}^{r} p_{i}^{\alpha_{i}}, \quad n=\prod_{j=1}^{s} q_{j}^{\beta_{j}}
$$

are prime decompositions for $m, n$. We use the following notations

$$
P=\prod_{i=1}^{r} p_{i}, \quad Q=\prod_{j=1}^{s} q_{j}, \quad \lambda(P, Q)=\prod_{r \mid \operatorname{lcm}(P, Q)}\left(1-\frac{1}{r}\right)
$$

(lcm means the least common multiple).

Theorem $2 J_{m, n} \geq 1$ if $\operatorname{gcd}(m, n)>\left[\prod_{i=1}^{r}\left(1+p_{i}\right)+\prod_{j=1}^{s}\left(1+q_{j}\right)\right](4 \lambda(P, Q))^{-1}$.

\footnotetext{
${ }^{\dagger}$ This work was supported by grants RFBR 05-01-01019 and NSh 1721.2003.1

1365-8050 (c) 2005 Discrete Mathematics and Theoretical Computer Science (DMTCS), Nancy, France
} 
The proofs of the theorems 1,2 are based on the following analytic and combinatorial results.

Let

$$
J_{N}(u)=\sum_{(a, N)=1} u^{a}, \quad N=p_{1}^{\alpha_{1}} \ldots p_{k}^{\alpha_{k}} .
$$

Lemma $1 J_{N}(u)=\frac{1}{1-u}-\sum_{i=1}^{k} \frac{1}{1-u^{p_{i}}}+\sum_{1 \leq i<j \leq k}^{k} \frac{1}{1-u^{p_{i} p_{j}}}-\ldots$

This formula can be easily proved by inclusion - exclusion principle.

Let $S_{r}(m, n)$ be the number of solutions of the system

$$
\begin{aligned}
& x+y=r, \\
& \operatorname{gcd}(x, m)=1, \operatorname{gcd}(y, n)=1
\end{aligned}
$$

The generating function for $S_{r}(m, n)$ is related with $J_{n}(u)$ by the following formula.

\section{Lemma 2}

$$
\sum_{r=1}^{\infty} S_{r}(m, n) u^{r}=J_{m}(u) J_{n}(u)
$$

Formula (3) implies an expression for the number of solutions of the system (1).

Lemma 3 Let $N=\operatorname{gcd}(m, n)+1$. Then the following equation holds

$$
\begin{aligned}
J_{m, n}=\operatorname{gcd}(m, n) & \sum_{u|P, v| Q} \frac{\mu(u) \mu(v)}{\operatorname{lcm}(u, v)}+\sum_{u|P, v| Q} \frac{\mu(u) \mu(v)(u+v)}{2 \operatorname{lcm}(u, v)}+ \\
& \sum_{u|P, v| Q} \frac{\mu(u)}{u} \sum_{\alpha^{u}=1} \frac{1}{\alpha^{N-1}\left(\alpha^{v}-1\right)}+\sum_{u|P, v| Q} \frac{\mu(v)}{v} \sum_{\alpha^{v}=1} \frac{1}{\alpha^{N-1}\left(\alpha^{u}-1\right)} .
\end{aligned}
$$

In sums of type

$$
\sum_{\alpha^{u}=1} \frac{1}{\alpha^{N-1}\left(\alpha^{v}-1\right)}
$$

the summation is over those roots of equation $\alpha^{u}=1$ that are not the roots of equation $\alpha^{v}=1$.

Sums (5) are called Dedekind sums. They are well-known in combinatorial analysis (e.g., see [3]).

To simplify (4) we use identities about Möbius function. They are 2-dimensional analogues of the classical formula

$$
\sum_{d \mid n} \frac{\mu(d)}{d}=\prod_{p \mid n}\left(1-\frac{1}{p}\right)
$$

An example of these identities is given by the following Lemma.

Lemma 4 ([4]) $\sum_{u|m, v| n} \frac{\mu(u) \mu(v)}{\operatorname{lcm}(u, v)}=\prod_{r \mid \operatorname{lcm}(P, Q)}\left(1-\frac{1}{r}\right)$. 
Dealing with Dedekind sums (5) we use the following useful statement. Let

$$
S_{n}(a)=\sum_{\alpha^{b}=1} \frac{1}{\alpha^{n}\left(\alpha^{a}-1\right)}
$$

where summation is over those roots of equation $x^{b}=1$ that are not the roots of equation $x^{a}=1$. By $m_{0}$ we denote the smallest positive solution of equation

$$
a x \equiv-(n+a) \quad(\bmod b) .
$$

Let $w(a, b)=m_{0}-1$.

Lemma 5

$$
S_{n}(a)=\frac{b}{2}-\frac{\operatorname{gcd}(a, b)}{2 \operatorname{lcm}(a, b)}-w(a, b)
$$

\section{References}

[1] Trotter W.T., Erdös P. When the cartesian product of directed cycles is hamiltonian. J. Graph Theory. V.2, 1978. P. $137-142$.

[2] Evdokimov A.A. Numeration of subsets of a finite set. (In Russian) Metody Diskret. Analiz. V. 34, 1980. P. 8-26.

[3] Ira M. Gessel. Generating functions and generalized Dedekind sums. The electronic Journal of Combinatorics. V. 4, no. 2, 1997.

[4] Leontiev V.K. Hamiltonian cycles in toric lattices. (In Russian) DAN, V. 395, no 5, 2004. P. 590-591. 
